\title{
Perinatal Audit
}

\section{Demographics}

1. Study code

2. Site

1=Tertiary hospital, 2=Quaternary hospital, 3=Stand-alone Maternity Unit

Choose one answer

3. Age

4. Country of birth

5. Geographical postcode

Choose one answer
1. Rural
2. Remote
3. Metropolitan
4. Unknown

6. Maternal race classification

Choose one answer

1. Caucasian

2. Aboriginal/Torres Strait Islander

3. Asian

4. Other:

7. Previous pregnancies with cardiac history?

Choose one answer

1. Yes

2. No previous pregnancies

3. Has had previous pregnancies but not with cardiac history

Details of previous pregnancy with cardiac history insert text

Follow-up for response of 'Yes'...

8. Weight

9. Height in $\mathrm{cm}$

10. Body mass index

\section{Current Social Status}

Choose one answer

1. Married

2. De facto/ living with partner

3. Divorced/Separated

4. Widowed

5. Never married/Single

\section{Cardiac pathology identified}

\section{Entry point}

Group A: Pre-existing cardiac condition, Group B: Newly acquired cardiac condition during course of pregnancy and so care may not be reflective of perinatal guidelines.

Choose one or more answers

1. Congenital (A)

2. Acquired A (acute event first trimester) (B)

3. Acquired B (at delivery) (B) 


\section{Perinatal Audit}

4. Acquired C (post-partum) (B)

5. Cyanotic $(\mathrm{A})$

6. Acyanotic $(\mathrm{A})$

7. Other:

13. Cause of cardiac disease

Choose one or more answers

1. Rheumatic heart disease (A)

2. Congenital heart disease (A)

3. Arrhythmias (B) (A)

4. Ischaemic Heart Disease (IHD) (B) (A)

5. Heart Failure (B) (A)

6. Cardiac arrest (B)

7. Conduction disorders (B)

8. PE (B)

9. Hypertension with severe cardiac condition (B)

10. Interventional coronary procedure (B)

11. Other (B) (A)

14. New York Heart Association (NYHA) Functional Classification on admission or Documented.

Applicable to category A conditions only Choose one or more answers

1. I

2. II

3. III

4. IV

5. Not applicable

6. Unknown

\section{Pre-conception care}

15. Documented discussion before conception, which is applicable to group A only. For all others leave as not applicable

Choose one answer
1. Yes
2. No
3. Not applicable
4. Unknown.

16. General physical assessment

Tick all which apply, Choose one or more answers
1. Weight
2. Height

3. BMI

4. Oral health

5. Respiratory examination (auscultation)

6. Breast Examination

7. Abdominal Examination

8. Vaginal examination (speculum/vaginal exam + swabs if clinically indicated)

9. Urinalysis

10. Psychosocial assessment

11. Not applicable

12. Unknown 


\section{Perinatal Audit}

17. Medication assessment:

Insert text

18. Cardiac assessment

Choose one or more answers

1. Blood pressure

2. Pulse

3. 12 lead ECG

4. Cardiac biomarkers

5. Cardiac auscultation

6. Echocardiogram

7. Not applicable

8. Unknown.

19. Cardiac assessment comments.

Insert text

20. Healthcare professionals who performed assessment

Choose one or more answers

1. Physician/medical officer

2. Midwife

3. Cardiac specialist nurse

4. Obstetrician

5. Not applicable

6. Unknown

7. Other:

21. Comments on healthcare professional who completed assessment Insert text

22. Hypertension management

Choose one answer

1. Not identified

2. Identified and management optimised/reviewed

3. Identified and management NOT optimised/reviewed

4. Not applicable

5. Unknown

23. Systolic blood pressure value:

24. Diastolic blood pressure value:

25. Arrhythmia management

Choose one answer

1. Not identified

2. Identified and management optimised/reviewed 


\section{Perinatal Audit}

3. Identified and management NOT optimised/reviewed

4. Not applicable

5. Unknown

26. Lifestyle factors identified

Choose one or more answers

1. Smoking

2. Exercise tolerance

3. Documented discussion with the woman, family and cardiologist re lifestyle factors

4. Psychosocial evaluation (identify tool used)

5. Not applicable

ANRQ score

Follow-up for response of 'Psychosocial evaluation (identify tool used) '...insert value and text EPPSD score

Follow-up for response of 'Psychosocial evaluation (identify tool used) '...insert value and text

\section{Preconception cardiac education}

27. Documentation of cardiac education

Choose one answer

1. Yes

2. No

3. Not applicable

28. Patient informed regarding the potential expected outcomes

Choose one or more answers

1. spontaneous miscarriage

2. live birth

3. death

4. antenatal care

5. anaesthesia

6. proposed care plan for pregnancy

7. medications in the Peripartum management

8. genetic counselling

9. Not applicable

10. Unknown

\section{Antenatal management}

29. Antenatal management offered

Choose one or more answers

1. Routine visit within $10-12$ weeks

2. Early ultrasound to establish estimated date of confirmation (EDC)

3. Documented multidisciplinary team management that includes: obstetrician,

4. cardiologist, obstetric physician, anaesthetist, intensivist 


\section{Perinatal Audit}

5. If applicable -fortnightly antenatal visits in high risk medical clinic dependant on

6. severity of disease.

7. Not applicable high risk antenatal visits

8. Avoid anaemia+ regular $\mathrm{Hb}$ checks.

9. Iron supplements + prenatal vitamins and dietary counselling

10. Anaesthetic review before 28 weeks

11. Unknown

\section{Clinical assessment}

30. Maternal age

31. Gestational age

32. Gravida

33. Parity

34. NYHA functional classification

Choose one answer

1. Documented

2. Not documented

3. Unknown

4. Not applicable

35. Comorbid conditions

Choose one or more answers

1. Previous cardiac events

2. Previous cardiac surgery

3. Previous cardiac interventions

4. Past/current cardiac lesions

5. Past/current cyanosis (Sa) $2<90 \%)$

6. Medication history

7. Use of cigarettes and/or alcohol

8. Not applicable

9. Unknown

36. Cardiac events additional information

Insert text

37. Cardiac surgery additional information

Insert text

38. Cardiac interventions additional information

Insert text

39. Past/current cardiac lesions additional information

Insert text

40. Past/current cyanosis additional information

Insert text

41. Medication history additional information 


\section{Perinatal Audit}

Insert text

42. Use of cigarettes and/or alcohol additional information

Insert text

\section{Clinical assessment: Investigations}

43. 12 lead ECG findings

44. Echocardiogram findings

45. Fetal wellbeing/risks

Choose one or more answers

1. Regular (fortnightly) ultrasound assessment of fetal wellbeing

2. Growth, umbilical artery Doppler, AFI

3. Cardiotocography (CTG) in third trimester

4. Unknown

5. Not applicable

46. Fetal risks

Choose one or more answers

1. Fetal echocardiogram at 20 weeks of gestation if woman on warfarin or identified cardiac fetal anomaly

2. Amniocentesis with antibiotic prophylaxis

3. Unknown

\section{Planned care}

\section{Echocardiogram assessment}

Choose one answer

1. Yes

2. No

3. Not applicable

4. Unknown

48. Ongoing NYHA functional classification to assist with hospital choice

Choose one answer

1. Yes

2. No

3. Not applicable

4. Unknown

49. Planned Care: Level of Hospital Care comments

50. Planned delivery mode

Choose one answer

1. Vaginal birth

2. Caesarean section for obstetric emergency only

3. Caesarean section for other

4. Not applicable

5. Unknown

51. Planned delivery mode comments

Insert 


\section{Perinatal Audit}

\section{High Risk management in labour}

52. Identified as high risk

Choose one answer
1. Yes
2. No
3. Not applicable
4. Unknown

53. Labour and birth occurred in a hospital with adult intensive care or cardiac unit Choose one answer
1. Yes
2. No
3. Not applicable

54. Documented plan of care in case-notes

Choose one answer
1. Yes
2. No
3. Unknown
4. Not applicable

55. Timing and labour of birth

Choose one answer

1. Multidisciplinary team discussion

2. Cardiac state well stabilised

3. At short notice in event of cardiac function and reserve altered

4. Unknown

5. Not applicable

56. During labour, the following multidisciplinary team members informed of impending birth and documented

Choose one or more answers

1. Cardiologist or physician

2. Anaesthetist

3. Paediatrician

4. Not applicable

5. Unknown

57. Appropriate antibiotic prophylaxis considered

Choose one answer
1. Yes
2. No
3. Not applicable
4. Unknown

58. Planned cardiac drugs required in consultation with multidisciplinary team Choose one answer
1. Yes
2. No
3. Not applicable
4. Unknown

59. High risk management in labour comments Insert text. 


\section{Perinatal Audit}

60. Was the second stage of birth shortened to avoid excessive maternal expulsive effort? Choose one or more answers
1. No
2. Yes with episiotomy
3. Yes with forceps delivery
4. Yes with no local anaesthetics with adrenaline being used
5. Not applicable
6. Unknown

61. Minimisation of cardiac compromise

Choose one or more answers

Consultation re avoiding the use of stirrups

- Assessment + prompt reporting of Tachycardia $>130 \mathrm{bpm}$

- Assessment + prompt reporting of Bradycardia $<40 \mathrm{bpm}$

- Assessment + prompt reporting of respiratory rate $>24$ or $<5 \mathrm{bpm}$

- Assessment+ prompt reporting rapidly changing pulse or respiration rate (even if

- within threshold )

- Not applicable

- Unknown

62. Birth comments

Insert text

63. Management in labour: Adult resuscitation equipment available?

Choose one answer

1. Yes

2. No

64. Management in labour: Ability to perform perimortem Caesarean section within 5 minutes of cardiac arrest

Choose one answer
1. Yes

2. No

65. Multidisciplinary team included in management of labour Choose one or more answers
1. Obstetrician
2. Midwife
3. Cardiologist
4. Physician/ medical officer
5. Anaesthetist
6. Intensivist
7. Other allied health professional
8. Other:

66. Management in labour comments

Insert text

67. Thromboembolism prophylaxis

Choose one or more answers

1. Use of anti- embolic stockings 


\section{Perinatal Audit}

2. Subcutaneous LMWH or IV Unfractionated heparin

3. Not applicable

4. Unknown

68. Pain Management and Anaesthesia

tick all which apply

Choose one or more answers

1. Anaesthetic consultation regarding appropriate pain management undertaken

2. Epidural considered

3. Combined spinal-epidural anaesthesia to decrease preload and afterload

4. Not applicable

5. Other:

69. Pain management and anaesthesia comments.

Insert text

\section{Preterm labour imminent}

70. Cardiac consultation with cardiologist and physician advice sought

Choose one answer

1. Yes

2. No

3. Not applicable

4. Unknown

71. Paediatric and neonatal staff available.

Choose one answer

1. Yes

2. No

3. Unknown

\section{Perinatal outcome of baby}

72. Outcome of birth

73. Gestational age

74. Live baby weight grams

75. Baby's length at birth

76. Baby's Head circumference at birth

77. Apgar score at 1 min Bounds: 0 - 10 [inclusive]

78. Apgar at 5 min Bounds: $0-10$ [inclusive]

79. Cardiac follow-up included in obstetric follow-up care

Checklist or nursing or medical documentation

Text Comments 